\title{
THE ARVESON BOUNDARY OF A FREE QUADRILATERAL IS GIVEN BY A NONCOMMUTATIVE VARIETY
}

\author{
ERIC EVERT
}

Abstract. Let $S M_{n}(\mathbb{R})^{g}$ denote the set of $g$-tuples of $n \times n$ real symmetric matrices and set $S M(\mathbb{R})^{g}=\cup_{n} S M_{n}(\mathbb{R})^{g}$. A free quadrilateral is the collection of tuples $X \in S M(\mathbb{R})^{2}$ which have positive semidefinite evaluation on the linear equations defining a classical quadrilateral. Such a set is closed under a generalized type of convex combination called a matrix convex combination. That is, given elements $X=\left(X_{1}, \ldots, X_{g}\right) \in S M_{n_{1}}(\mathbb{R})^{g}$ and $Y=\left(Y_{1}, \ldots, Y_{g}\right) \in S M_{n_{2}}(\mathbb{R})^{g}$ of a free quadrilateral $\mathscr{Q}$, one has

$$
V_{1}^{T} X V_{1}+V_{2}^{T} Y V_{2} \in \mathscr{Q}
$$

for any contractions $V_{1}: \mathbb{R}^{n} \rightarrow \mathbb{R}^{n_{1}}$ and $V_{2}: \mathbb{R}^{n} \rightarrow \mathbb{R}^{n_{2}}$ satisfying $V_{1}^{T} V_{1}+V_{2}^{T} V_{2}=I_{n}$. These matrix convex combinations are a natural analogue of convex combinations in the dimension free setting.

Elements of a free quadrilateral which cannot be expressed as a nontrivial matrix convex combination of other elements of the free quadrilateral are called free extreme points. Free extreme points serve as the minimal set which recovers a free quadrilateral through matrix convex combinations. In this way, free extreme points are the natural type of extreme point for a free quadrilateral.

In this article we show that the set of free extreme points of a free quadrilateral is determined by the zero set of a collection of noncommutative polynomials. More precisely, given a free quadrilateral $\mathscr{Q}$, we construct noncommutative polynomials $p_{1}, p_{2}, p_{3}, p_{4}$ such that a tuple $X \in S M(\mathbb{R})^{2}$ is a free extreme point of $\mathscr{Q}$ if and only if $X \in \mathscr{Q}$ and $p_{i}(X)=0$ for $i=1,2,3,4$ and $X$ is irreducible.

In addition, we establish several basic results for projective maps of free spectrahedra and for homogeneous free spectrahedra. In particular, we show that that the image of a free extreme point under an invertible projective map is again a free extreme point. We also extend a kernel condition for a tuple to be a free extreme point to the setting of homogeneous free spectrahedra.

Mathematics subject classification (2020): Primary 47L07; Secondary 46L07, 90C05.

Keywords and phrases: Matrix convex set, extreme point, Arveson boundary, linear matrix inequality (LMI), spectrahedron, free linear programming.

\section{REFERENCES}

[1] J. AgLER, An abstract approach to model theory, Surveys of some recent results in operator theory, Vol. II, 1-23, Pitman Res. Notes Math. Ser., 192, Longman Sci. Tech., Harlow, 1988.

[2] J. AgLeR, J. E. MCCARTHY, Global holomorphic functions in several non-commuting variables, Canad. J. Math. 67 (2015) 241-285.

[3] W. Arveson, Subalgebras of $C^{*}$-algebras, Acta Math. 123 (1969) 141-224.

[4] W. ARveson, Subalgebras of C* -algebras, II, Acta Math. 128 (1972) 271-308.

[5] W. Arveson, The noncommutative Choquet boundary, J. Amer. Math. Soc. 21 (2008) 1065-1084.

[6] J. A. BALL, V. Bolotnikov, Interpolation in the noncommutative Schur-Agler class, J. Operator Theory 58 (2007) 83-126.

[7] K. R. Davidson, M. Kennedy, The Choquet boundary of an operator system, Duke Math. J. 164 (2015) 2989-3004. 
[8] K. R. Davidson, M. Kennedy, Noncommutative Choquet Theory, preprint https://arxiv.org/pdf/1905.08436.pdf.

[9] K. R. Davidson, A. Dor-On, O. Shalit, B. Solel, Dilations, inclusions of matrix convex sets, and completely positive maps, Int. Math. Res. Not. 13 (2017) 4069-4130.

[10] M. A. Dritschel, S. A. MCCullough, Boundary representations for families of representations of operator algebras and spaces, J. Operator Theory 53 (2005) 159-168.

[11] E. G. EFFROS, S. WINKLER, Matrix convexity: operator analogues of the bipolar and Hahn-Banach theorems, J. Funct. Anal. 144 (1997) 117-152.

[12] E. EVERT, Matrix convex sets without absolute extreme points, Linear Algebra Appl. 537 (2018) 287301.

[13] E. Evert, J. W. Helton, Arveson extreme points span free spectrahedra, Math. Ann. 375, 629-653 (2019), https://doi.org/10.1007/s00208-019-01858-9.

[14] E. Evert, J. W. Helton, I. Klep, S. McCullough, Extreme points of matrix convex sets, free spectrahedra and dilation theory, J. of Geom. Anal. 28 (2018) 1373-1498.

[15] D. R. FARENICK, Extremal matrix states on operator systems, J. London Math. Soc. 61 (2000) 885892.

[16] D. R. FARENICK, Pure matrix states on operator systems, Linear Algebra Appl. 393 (2004) 149-173.

[17] T. Fritz, T. Netzer, A. Thom, Spectrahedral Containment and Operator Systems with Finitedimensional Realization, SIAM J. Appl. Algebra Geom. 1 (2017) 556-574.

[18] A. H. FUller, M. HARTZ, M. LUPINI, Boundary representations of operator spaces, and compact rectangular matrix convex sets, J. Operator Theory 79 (2018) 139-172.

[19] M. Hamana, Injective envelopes of operator systems, Publ. Res. Inst. Math. Sci. 15 (1979) 773-785.

[20] J. W. Helton, I. KleP, S. MCCullough, The matricial relaxation of a linear matrix inequality, Math. Program. 138 (2013) 401-445.

[21] J. W. Helton, I. Klep, S. McCullough, The tracial Hahn-Banach theorem, polar duals, matrix convex sets, and projections of free spectrahedra, J. Eur. Math. Soc. 6 (2017) 1845-1897.

[22] J. W. Helton, I. KleP, J. VolČIČ, Factorization of noncommutative polynomials and nullstellensätze for the free algebra, Int. Math. Res. Not. (2020), https://doi.org/10.1093/imrn/rnaa122.

[23] J. W. Helton, S. MCCullough, Every free basic convex semi-algebraic set has an LMI representation, Ann. of Math. (2) 176 (2012) 979-1013.

[24] M. Jury, I. Klep, M. Mancuso, S. McCullough, J. E. Pascoe, Noncommutative Partial Convexity Via $\Gamma$-Convexity, J. of Geom. Anal. (2020), https://doi.org/10.1007/s12220-020-00387-1.

[25] D. KALYUZHNYI-VERBOVETSKIĬ, V. VINNIKOV, Foundations of free noncommutative function theory, Amer. Math. Soc., 2014.

[26] C. KLESKI, Boundary representations and pure completely positive maps, J. Operator Theory 71 (2014) 45-62.

[27] T. L. Kriel, An Introduction to Matrix Convex Sets and Free Spectrahedra, Complex Anal. Oper. Theory 13 (2019) 3251-3335, https: //doi .org/10.1007/s11785-019-00937-8.

[28] P. S. Muhly, B. Solel, Progress in noncommutative function theory, Sci. China Ser. A 54 (2011) 2275-2294.

[29] P. S. Muhly, B. Solel, An algebraic characterization of boundary representations, Nonselfadjoint operator algebras, operator theory, and related topics, 189-196, Oper. Theory Adv. Appl., 104, Birkhäuser, Basel, 1998.

[30] B. Passer, V. PAUlsen, Matrix range characterizations of operator system properties, preprint https://arxiv.org/pdf/1912.06279.pdf.

[31] B. Passer, O. Shalit, B. Solel, Minimal and maximal matrix convex sets, J. Funct. Anal. 274 (2018) 3197-3253.

[32] G. POPESCU, Free holomorphic automorphisms of the unit ball of $B(H)^{n}$, J. reine angew. Math. 638 (2010) 119-168.

[33] G. Salomon, O. M. Shalit, E. Shamovich, Algebras of bounded noncommutative analytic functions on subvarieties of the noncommutative unit ball, Trans. Amer. Math. Soc. 370 (2018) 8639-8690.

[34] D.-V. Voiculescu, Free analysis questions II: The Grassmannian completion and the series expansions at the origin, J. reine angew. Math. 645 (2010) 155-236. 
[35] C. Webster, S. Winkler, The Krein-Milman theorem in operator convexity, Trans. Amer. Math. Soc. 351 (1999) 307-322.

[36] A. ZALAR, Operator Positivstellensätze for noncommutative polynomials positive on matrix convex sets, J. Math. Anal. Appl. 445 (2017) 32-80. 